\title{
Effect of co-culturing human primary basic fibroblasts with respiratory syncytial virus- infected 16-HBE cells
}

\author{
J.-H. Wang, L.-H. Sun, S.-K. Huang and A.-H. Chen \\ Guangzhou Panyu Central Hospital, \\ The First Affiliated Hospital of Guangzhou Medical College, Guangzhou, China \\ Corresponding author: A.-H. Chen \\ E-mail: chenaihua_ah@163.com \\ Genet. Mol. Res. 15 (1): gmr.15017339 \\ Received July 29, 2015 \\ Accepted October 19, 2015 \\ Published February 5, 2016 \\ DOI http://dx.doi.org/10.4238/gmr.15017339
}

ABSTRACT. This study investigated the effects induced by co-culturing human primary basic fibroblasts (HPBFs) with 16-human bronchial epithelial cells (16-HBE) infected with respiratory syncytial virus (RSV), in particular the transformation of HPBFs into myofibroblasts and secretion of extracellular matrix proteins. HPBFs were co-cultured with 16-HBE cells infected with RSV and quantitatively analyzed. We constructed models of HPBFs co-cultured with 16-HBE cells that were either uninfected (control group) or infected with RSV (experimental group). Following initiation of cocultures, HPBFs and supernatants were collected at 24-h intervals up to 120 h. Expression of $\alpha$-smooth muscle actin ( $\alpha-S M A)$ was detected by indirect immunofluorescence and western blotting, while type I collagen (Col I) and fibronectin were analyzed by competitive enzyme-linked immunosorbent assays. After $72 \mathrm{~h}$, $\alpha$-SMA expression increased in HPBFs cultured with RSV-infected 16-HBE relative to uninfected controls, reaching its highest level at $96 \mathrm{~h}$. Similarly, Col I secretion was also higher in HPBFs co-cultured with RSV-infected 16-HBE relative to uninfected controls; Col I secretion increased with time and reached its highest level at $120 \mathrm{~h}$. HPBFs were transformed into myofibroblasts following co-culture with RSV-infected 16- 
HBE, which when combined with the observed increase in Col I secretion suggests that airway remodeling would then be promoted.

Key words: Primary basic fibroblast; Bronchial epithelial cells; Respiratory syncytial virus; a-SMA; Type I collagen; Fibronectin

\section{INTRODUCTION}

Respiratory syncytial virus (RSV) is an important factor leading to respiratory tract infection in infants. In the early stages of infection, lower respiratory tract infections are closely related to asthma pathogenesis. Studies in rat models have shown that RSV infection not only induces acute airway inflammation and blockage, but also leads to chronic airway inflammation and bronchial hyper-responsiveness (BHR), which can persist for 5 months without the detection of RSV (Jafri et al., 2004). Becnel et al. (2005) found that RSV infection of ovalbumin-sensitized mice results in chronic airway inflammation and BHR, while inducing airway epithelium thickening and increased deposition of fibrosis and collagen, which leads to airway remodeling. Clinical follow-ups of children diagnosed with RSV-induced bronchiolitis as infants indicated that FEV1/FVC and FEF75 levels were lower than those observed in controls, indicating that RSV infection could lead to histopathological changes in those with RSV-induced bronchiolitis (Sigurs et al., 2005). Therefore, airway remodeling may occur when RSV infection induces acute asthma, but the mechanisms by which this happens are still unclear. In our study, we constructed a model of RSV infection by coculturing human primary basic fibroblasts cells (HPBFs) with 16-human bronchial epithelial cells (16-HBE) that had been infected with RSV in vitro. We used this model to investigate the process by which HPBFs transform into myofibroblasts, the effect on secretion of extracellular matrix (ECM) proteins, and the mechanism of airway remodeling. Our results provide a theoretical basis for the prevention and treatment of persistent asthma after RSV infection.

\section{MATERIAL AND METHODS}

\section{Cells, viruses, and reagents}

RSV-A2 was obtained from American Type Culture Collection (Washington, USA). HEp-2 cells were obtained from Shanghai Institutes for Biological Sciences (Chinese Academy of Science, Shanghai, China). 16-HBE cells were obtained from University of Southampton (Southampton, UK). MEM and DMEM/F12 were obtained from Gibco, Thermo Fisher Scientific Inc. (Shanghai, China). Fetal bovine serum (FBS) was obtained from HangZhou SIJIQING Bioengineering Materials Company (HangZhou, China). Culture dishes, mouse anti-human $\alpha$-SMA monoclonal antibody and PVDF membranes were obtained from Millipore (Billerica, MA, USA). Collagen I (Col I) ELISA kits were obtained from Aquatic Diagnostics, Ltd. (Beijing, China). Mouse anti-human $\beta$-actin monoclonal antibodies were obtained from Thermo Fisher Scientific, Inc. Goat anti-mouse secondary antibodies were obtained from Southern Biotechnology Associates (Birmingham, USA). FITC-conjugated goat anti-mouse secondary antibodies were obtained from WuHan Boster Bioengineering Company, Ltd. (WuHan, China).

\section{HPBF culturing}

Adjacent normal and cancerous bronchial tissue samples were obtained from lung cancer 
patients hospitalized at the Department of Thoracic Surgery of the First Affiliated Hospital of Guangzhou Medical University. The epithelial layer of each sample was removed and discarded, and tissues were cut into $1-2-\mathrm{mm}^{3}$ cubes and incubated at $37^{\circ} \mathrm{C}$ in bottles containing $2 \mathrm{~mL} \mathrm{DMEM} /$ F12 supplemented with $10 \%$ FBS. When cell cultures reached $90 \%$ confluence, tissues were removed, attached to blocks, and cultured in separate bottles. Cells were cultured through 1-3 passages, then immunolabeled with rabbit anti-human vimentin monoclonal antibodies.

\section{HEp-2 cell culture and quantification of RSV}

HEp-2 cells were cultured through multiple passages and cryopreserved as previously described (McKimm-Breschkin, 2004). To analyze virus titers, a plaque formation test was utilized as follows. HEp-2 cells were cultured on 24 -well dishes to $100 \%$ confluence. Viral suspensions were diluted 1:10 with DMEM/F12 containig 10\% FBS and added to wells (30 $\mu \mathrm{L}$ per well) through three parallel holes in lids. Uninfected cells were used as controls. Following $2 \mathrm{~h}$ of virus adsorption, DMEM containing 15\% carboxymethyl cellulose and 2\% FBS (CMC-DMEM) was added, $0.5 \mathrm{~mL}$ of each well. After 5 days, CMC-DMEM medium was discarded, and cells were simultaneously fixed and stained in PBS containing $0.1 \%$ crystal violet and $10 \%$ formaldehyde, at $25^{\circ} \mathrm{C}$. Virus titers were calculated by counting the number of plaques, according to the following equation: $P F U=a, b / v$, where PFU is the number of virus plaques, $a$ is the average number of plaques, $b$ is the reciprocal of the virus dilution rate, and $v$ is the volume of virus in each well. Multiplicity of infection (MOI) was calculated as the volume of virus $(\mathrm{mL}) \times \mathrm{PFU} /$ number of cells.

\section{RSV infection of 16-HBE}

16-HBE cells were seeded on cover glass slides at $1 \times 10^{5}$ cells per well. After $2 \mathrm{~h}$ of virus adsorption $(\mathrm{MOI}=0.1)$, medium containing viruses was discarded, and cells were cultured for 72 $\mathrm{h}$ at $25^{\circ} \mathrm{C}$ in DMEM containing $2 \% \mathrm{FBS}$. Glass slides were removed and fixed in acetone, at $25^{\circ} \mathrm{C}$, and fluorescence detection was carried out with monoclonal antibodies.

\section{Co-culture of RSV-infected 16-HBE with HPBFs}

HPBFs were seeded on 6-well dishes at $5 \times 10^{5}$ cells per well. Experiments included two groups, one with uninfected 16-HBE seeded on slides and one with RSV-infected 16-HBE seeded on slides as described above, all the 16-HBE. HPBFs and supernatants were collected after 24, $48,72,96$, and $120 \mathrm{~h}$.

\section{$\alpha-S M A$ expression analysis by immunofluorescence}

Slides were placed on 24-well dishes, and cells were fixed for $10 \mathrm{~min}$ in $10 \%$ formaldehyde. Fixed cells were then washed 3 times in PBS, then permeabilized in $0.5 \%$ Triton X-100 for 10 min. Cells were washed in PBS, blocked in $0.5 \%$ bovine serum albumin for $20 \mathrm{~min}$, then incubated in mouse anti-human $\alpha$-SMA monoclonal antibody (1:100) overnight at $4^{\circ} \mathrm{C}$. Cells were washed 3 times in PBS, incubated in FITC-conjugated goat anti-mouse secondary antibodies for $45 \mathrm{~min}$ at room temperature, then washed 3 times in PBS. Finally, cells were stained with Hoechst for 10 min, then sealed onto slides in $80 \%$ glycerol and observed by fluorescence microscopy. 


\section{a-SMA expression analysis by western blotting}

Cells were digested with trypsin and collected, then centrifuged at $3000 \mathrm{~g}$ for $10 \mathrm{~min}$. Supernatants were discarded, while pellets were washed twice in PBS $\left(4^{\circ} \mathrm{C}\right)$, and then lysed by addition of $200 \mu \mathrm{L}$ lysis buffer ( $1 \mathrm{~mL}$ RIPA buffer containing $10 \mu \mathrm{L}$ PMSF). Lysates were placed on ice for $30 \mathrm{~min}$, then centrifuged at $12,000 \mathrm{~g}$ for $5 \mathrm{~min}$ at $4^{\circ} \mathrm{C}$; supernatants were removed and stored at $-70^{\circ} \mathrm{C}$. Total protein contents were quantified by BCA assay (BCA kit, Beyotime Biotechnology, Shanghai, China). Samples were separated by SDS-PAGE (10\% separation gel, $4 \%$ stacking gel, $40 \mathrm{mg}$ protein per well), then transferred to polyvinylidene fluoride membranes (Millipore Corporation) at $350 \mathrm{~mA}$ for $90 \mathrm{~min}$. Following transfer, membranes were blocked in 5\% non-fat milk for $1.5 \mathrm{~h}$ at room temperature, then incubated overnight in anti- $\alpha$-SMA and anti- $\beta$-actin primary antibodies $(1: 1000)$ at $4^{\circ} \mathrm{C}$. Following washes in PBS, blots were incubated in goat antimouse secondary antibodies $(1: 4000)$ for $1.5 \mathrm{~h}$ at room temperature. The blots were exposed with ECL system on films by a DLK-14 Delight machine (Delight, Suzhou, China), and the Bio-Rad image analysis software was used to analyze proteins.

\section{Examination of Col I and fibronectin (Fn)}

Col I and Fn were analyzed by ELISA and stored at $-80^{\circ} \mathrm{C}$.

\section{Statistical analysis}

All data were analyzed by the SPSS 16.0 software. Data measurements are reported as means \pm standard deviation. Comparison of multiple groups was analyzed by one-way ANOVA, while $t$-tests were utilized to compare data between groups; $P$ values $<0.05$ were considered significant.

\section{RESULTS}

\section{Identification of HPBFs}

Fibroblasts labeled with rabbit anti-human vimentin monoclonal antibodies were detected by brownish yellow staining, as shown in Figure 1.

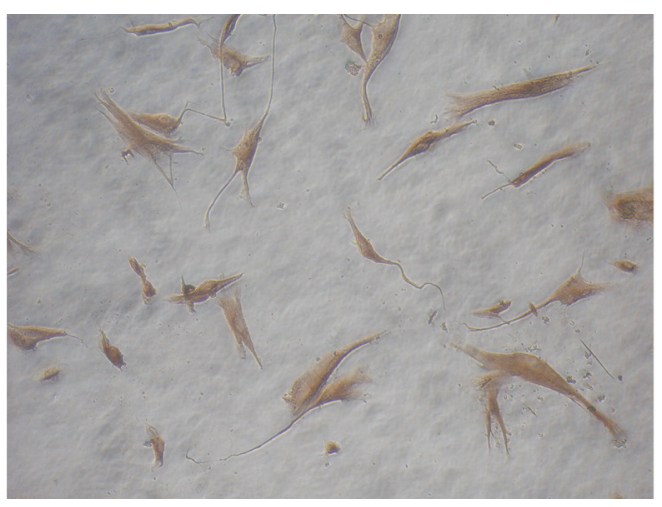

Figure 1. Vimentin staining of fibroblasts. 


\section{Detection of RSV antigen in 16-HBE by immunofluorescence}

Green fluorescence was detected in the cytoplasm of 16-HBE after $72 \mathrm{~h}$ of RSV infection, which indicated that RSV was mainly distributed in the cytoplasm (Figure 2). Detection of virus titer by plaque formation experiment is shown in Figure 3.

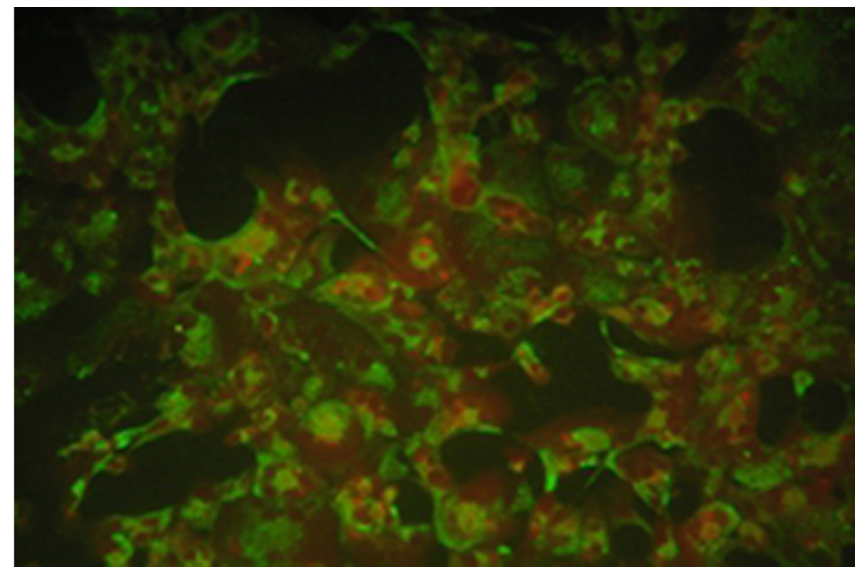

Figure 2. Green fluorescence expression of RSV-specific antigen in 16-HBE cells after $72 \mathrm{~h}$ of RSV infection.

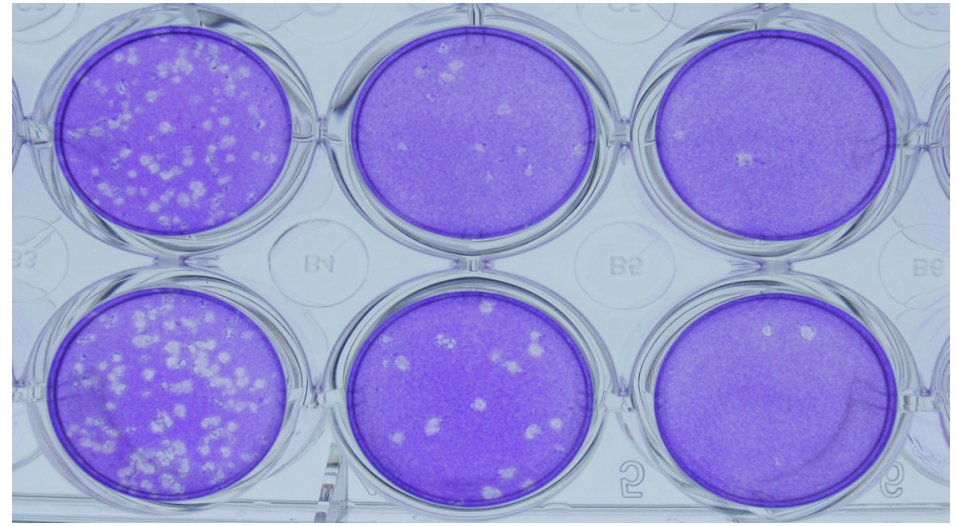

Figure 3. Plaque formation after RSV infection of HEp-2 cells.

\section{a-SMA expression in HPBFs following co-culture with RSV-infected 16-HBE}

\section{Immunofluorescence detection of a-SMA expression in HPBFs}

After $24 \mathrm{~h}$, there was no significant $\alpha$-SMA expression in HPBFs co-cultured with either uninfected or RSV-infected 16-HBE. However, after $48 \mathrm{~h}$, greater expression of $\alpha$-SMA in HPBFs was observed in co-cultures with RSV-infected 16-HBE, which reached its highest level at $96 \mathrm{~h}$ (Figure 4). 


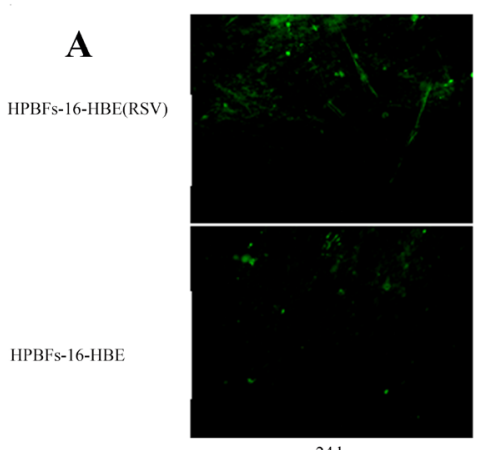

$24 \mathrm{~h}$
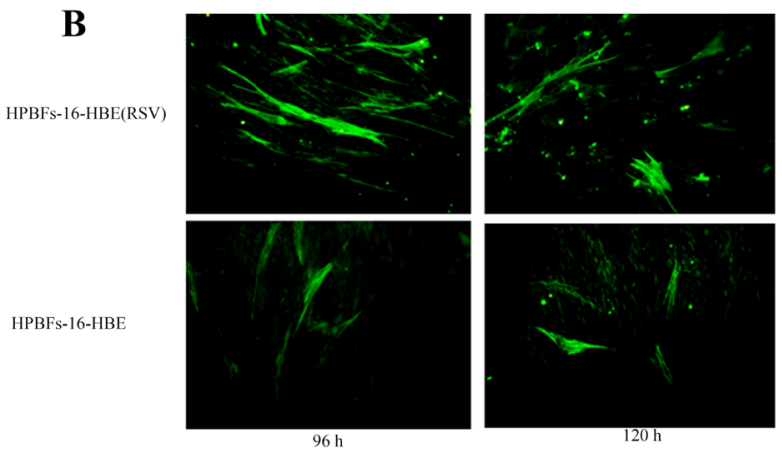

Figure 4. Effect of RSV infection of 16-HBE cells on $\alpha$-SMA expression in HPBFs, as assayed by immunofluorescence.

\section{a-SMA expression analysis by western blotting}

After 24 and $48 \mathrm{~h}$, western blotting could detect no significant differences in $\alpha$-SMA expression in HPBFs co-cultured with either uninfected or RSV-infected 16-HBE cells. From 72 $\mathrm{h}$ onward, different levels of a-SMA expression were observed between these two groups, which for the virus-infected group [HPBFs-16-HBE (RSV)] reached its highest level at $96 \mathrm{~h}$ (Figure 5). Significant differences were observed between the $72 \mathrm{~h}(t=25.66)$ and $120 \mathrm{~h}(t=80.974)$ time points $(P<0.05)$. In the uninfected group (HPBFs-16-HBE), $\alpha$-SMA expression was higher at 96 $\mathrm{h}$ than at $72 \mathrm{~h}(t=22.6)$ and $120 \mathrm{~h}(t=16.507)(\mathrm{P}<0.05)$. In the HPBFs-16-HBE (RSV) group, a-SMA expression at 72, 96, and $120 \mathrm{~h}$ were higher than levels observed in the HPBFs-16-HBE group (Table 1), which indicated that RSV infection of 16-HBE could promote HPBF transformation into myofibroblasts.

\section{Effect on Col I and Fn in HPBFs co-cultured with RSV-infected 16-HBE}

Col I secretion levels in the HPBFs-16-HBE (RSV) group were significantly higher than those of the HPBFs-16-HBE group at all observed time points (24-120 h) (Table 2). Col I secretion levels increased gradually in this group, and reached their highest level at $120 \mathrm{~h}$, which was significantly higher than at $24 \mathrm{~h}(t=61.776), 48 \mathrm{~h}(t=40.876), 72 \mathrm{~h}(t=54.11)$, and $96 \mathrm{~h}(t=33.845)$. 


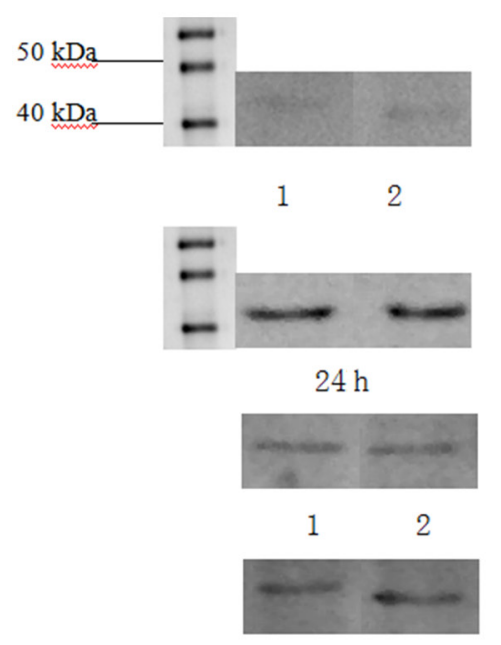

$96 \mathrm{~h}$

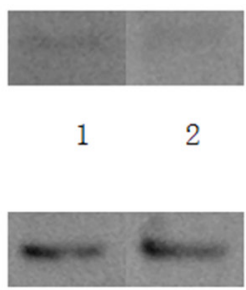

$48 \mathrm{~h}$

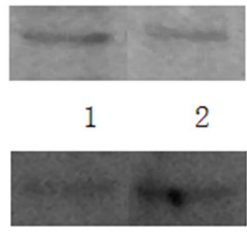

$120 \mathrm{~h}$

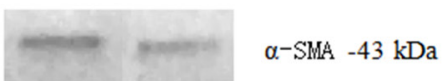

12

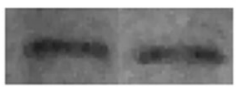

$\beta-\operatorname{actin}-42 \mathrm{kDa}$

$-\mathrm{SMA}-43 \mathrm{kDa}$

$\alpha-\mathrm{SMA}-43 \mathrm{kDa}$

$\beta-\operatorname{actin}-42 \mathrm{kDa}$

Figure 5. Effect of RSV infection of 16-HBE cells on $\alpha$-SMA expression in HPBFs, as assayed by western blotting. For each sample, lane 1 = HPBFs-16-HBE (RSV), lane 2 = HPBFs-16-HBE (uninfected).

Table 1. $\alpha-S M A$ expression in HPBFs after RSV infection of 16-HBE ( $\alpha-S M A / \beta-a c t i n)$.

\begin{tabular}{l|c|c|c|c|c|c|c|c|c}
\hline Group & $72 \mathrm{~h}$ & $t^{\mathrm{a}}$ & $\mathrm{P}$ & $96 \mathrm{~h}$ & $t^{\mathrm{a}}$ & $\mathrm{P}$ & $120 \mathrm{~h}$ & $t^{\mathrm{a}}$ & $\mathrm{P}$ \\
\hline HPBFs-16-HBE & $0.434 \pm 0.023$ & & & $0.825 \pm 0.019$ & & & $0.631 \pm 0.01$ & & \\
\hline HPBFs-16-HBE (RSV) & $0.482 \pm 0.018$ & 21.883 & 0.002 & $0.857 \pm 0.018$ & 61.836 & 0.000 & $0.675 \pm 0.012$ & 4.826 & 0.04 \\
\hline
\end{tabular}

aComparing to group HPBFs-16-HBE

Table 2. Col I secretion level in HPBFs after RSV infection of 16-HBE (means \pm SD).

\begin{tabular}{l|c|c|c|c|c}
\hline Group & $24 \mathrm{~h}$ & $48 \mathrm{~h}$ & $72 \mathrm{~h}$ & $96 \mathrm{~h}$ & $120 \mathrm{~h}$ \\
\hline HPBFs-16-HBE & $27.89 \pm 9.62$ & $32.73 \pm 2.78$ & $40.98 \pm 2.22$ & $54.07 \pm 2.96$ & $76.05 \pm 4.44$ \\
\hline HPBFs-16-HBE-RSV & $96.73 \pm 1.85$ & $118.98 \pm 2.22$ & $138.35 \pm 5.18$ & $155.37 \pm 6.29$ & $180.76 \pm 1.48$ \\
\hline$t$ & 14.694 & 380.474 & 32.216 & 28.812 & 43.301 \\
\hline $\mathrm{P}$ & 0.005 & 0.000 & 0.001 & 0.001 & 0.001 \\
\hline
\end{tabular}

There were no significant differences in Fn secretion levels between the HPBFs-16-HBE (RSV) and HPBFs-16-HBE groups at any observed time points (Table 3).

Table 3. Fn secretion level in HPBFs after RSV infection of $16-\mathrm{HBE}$ (means \pm SD).

\begin{tabular}{l|c|c|c|c|c|c|c}
\hline Group & $24 \mathrm{~h}$ & $48 \mathrm{~h}$ & $72 \mathrm{~h}$ & $96 \mathrm{~h}$ & $120 \mathrm{~h}$ & $\mathrm{~F}$ & $\mathrm{P}$ \\
\hline HPBFs-16-HBE & $18.98 \pm 0.11$ & $18.76 \pm 0.48$ & $18.90 \pm 0.21$ & $18.73 \pm 0.57$ & $19.07 \pm 1.03$ & 0.366 & 0.827 \\
\hline HPBFs-16-HBE-RSV & $18.76 \pm 0.40$ & $18.68 \pm 0.43$ & $18.32 \pm 0.53$ & $18.52 \pm 0.53$ & $18.36 \pm 0.60$ & 0.882 & 0.509 \\
\hline$t$ & 1.058 & 4.157 & 1.934 & 0.467 & 1.062 & & \\
\hline $\mathrm{P}$ & 0.401 & 0.053 & 0.193 & 0.686 & 0.4 & & \\
\hline
\end{tabular}

These results showed that RSV infection of 16-HBE could promote HPBFs to transform into myofibroblasts, while increasing the secretion of $\mathrm{Col} I$ and thereby the synthesis of ECM, which promotes airway remodeling. 


\section{DISCUSSION}

At early stages of child development, viral infection is closely related to asthma, and infection of the respiratory tract by RSV is very significant in infant medical care. Almost all infants are infected by RSV in the two years following birth (Gardner and McQuillin, 1968). Sigurs et al. (2005) found that the recurrent wheezing incidence and asthma diagnosis rate of those children who had RSV bronchiolitis as infants were significantly higher than in uninfected individuals. In a study of more than 1000 children, Stein et al. (1999) found that the recurrent wheezing incidence of those who had RSV lower respiratory tract infection (RSV-LRTI) before age 3 was significantly higher than those who had not experienced RSV-LRTI. Given that the respiratory systems of children are in a stage of rapid development and maturation, these studies underscore that children are highly susceptible to induced chronic airway histopathology changes, especially those children who experienced RSV-LRTI as infants. These studies thereby provided the pathophysiological basis for following reactive airway disease and chronic asthma.

By studying animal models, many scientists have found that RSV infection can lead to chronic airway inflammation, BHR, and airway remodeling. Jafri et al. (2004) found that RSV infection led to not only acute airway inflammation and blockage, but also chronic airway inflammation and BHR in rats, which persisted for 5 months after RSV was no longer detected in the lungs. Miller et al. (2002) and Mejías et al. (2005) also found that RSV infection could lead to chronic airway inflammation in rats; however, after removal of airway inflammation BHR was not promoted, indicating that this effect is less dependent on airway inflammation than may have been believed. Previous studies have indicated that airway inflammation is the main pathological basis for BHR, whereas the present study has shown that airway remodeling is also an important mechanism of BHR. The characteristics of airway remodeling included the thickening of reticular basement membranes, subepithelial fibrosis, hyperplasia and hypertrophy of myofibroblasts and smooth muscle cells, hyperplasia of mucus, and microvascular generation (Stein et al., 1999). The thickening of reticular basement membranes is mainly induced by sedimentation of Col I and Col III, mucins, fibronectin, and other ECM factors (Miller et al., 2002). These proteins are secreted by active myofibroblasts, which are the transitional cell from fibroblasts to smooth muscle cells. Compared to fibroblasts, $\alpha$-SMA expression and the synthesis and secretion of many cytokines and ECM proteins are increased in myofibroblasts (Mejías et al., 2005). Thickening of airway walls induced by airway remodeling, and an increase in airway myofibroblasts and smooth muscle cells can lead to obvious stenosis with slight stimulation, increasing resistance, and significant BHR; the two studies mentioned above indicated that RSV-LRTI infection can lead to airway remodeling, which might result in a similar array of symptoms.

Becnel et al. (2005), Tourdot et al. (2008) and Vignola et al. (2003) found that RSV infection of ovalbumin-sensitized mice leads to chronic airway inflammation and BHR, along with thickening of airway subepithelial lamellae, hyperplasia and hypertrophy of airway smooth muscle cells, subepithelial fibrosis, and increased collagen deposition. By examination of pulmonary ventilation function, Sigurs et al. (2005) found that children who experienced RSV-LRTI demonstrated lower FEV1/FVC and PEF75 levels than controls. This suggested that RSV infection of airway epithelial cells co-cultured with HPBFs could promote HPBF transformation into myofibroblasts and increased secretion of Col I (both of which were observed in our study), which could then lead to airway remodeling and BHR.

In conclusion, when RSV-LRTI induces acute asthma, airway remodeling can be 
activated, which is a basis for the pathophysiological changes induced by reactive airway disease and persistent asthma. However, the mechanisms of RSV infection and airway remodeling are complicated, including the regulation of many interactions between cells by cytokines. Further investigation is needed to provide more details of the mechanisms by which these processes occur.

\section{REFERENCES}

Becnel D, You D, Erskin J, Dimina DM, et al. (2005). A role for airway remodeling during respiratory syncytial virus infection. Respir. Res. 6: 122. http://dx.doi.org/10.1186/1465-9921-6-122

Gardner PS and McQuillin J (1968). Application of immunofluorescent antibody technique in rapid diagnosis of respiratory syncytial virus infection. Br. Med. J. 3: 340-343. http://dx.doi.org/10.1136/bmj.3.5614.340

Jafri HS, Chavez-Bueno S, Mejias A, Gomez AM, et al. (2004). Respiratory syncytial virus induces pneumonia, cytokine response, airway obstruction, and chronic inflammatory infiltrates associated with long-term airway hyperresponsiveness in mice. J. Infect. Dis. 189: 1856-1865. http://dx.doi.org/10.1086/386372

McKimm-Breschkin JL (2004). A simplified plaque assay for respiratory syncytial virus - direct visualization of plaques without immunostaining. J. Virol. Methods 120: 113-117. http://dx.doi.org/10.1016/j.jviromet.2004.02.020

Mejías A, Chavez-Bueno S, Jafri HS and Ramilo O (2005). Respiratory syncytial virus infections: old challenges and new opportunities. Pediatr. Infect. Dis. J. 24: S189-S197. http://dx.doi.org/10.1097/01.inf.0000188196.87969.9a

Miller M, Cho JY, Baek KJ, Castaneda D, et al. (2002). Plasmid DNA encoding the respiratory syncytial virus G protein protects against RSV-induced airway hyperresponsiveness. Vaccine 20: 3023-3033. http://dx.doi.org/10.1016/S0264410X(02)00217-7

Sigurs N, Gustafsson PM, Bjarnason R, Lundberg F, et al. (2005). Severe respiratory syncytial virus bronchiolitis in infancy and asthma and allergy at age 13. Am. J. Respir. Crit. Care Med. 171: 137-141. http://dx.doi.org/10.1164/rccm.200406$\underline{7300 \mathrm{C}}$

Stein RT, Sherrill D, Morgan WJ, Holberg CJ, et al. (1999) Respiratory syncytial virus in early life and risk of wheeze and allergy by age 13 years. Lancet 354: 541-545. http://dx.doi.org/10.1016/S0140-6736(98)10321-5

Tourdot S, Mathie S, Hussell T, Edwards L, et al. (2008). Respiratory syncytial virus infection provokes airway remodelling in allergen-exposed mice in absence of prior allergen sensitization. Clin. Exp. Allergy 38: 1016-1024. http://dx.doi. org/10.1111/j.1365-2222.2008.02974.x

Vignola AM, Mirabella F, Costanzo G, Di Giorgi R, et al. (2003). Airway remodeling in asthma. Chest 123: 417S-422S. http:// dx.doi.org/10.1378/chest.123.3 suppl.417S 